Portland State University

PDXScholar

7-31-1975

\title{
A Conditioning Model for the McCollough Effect
}

Andreas D. Lord

Portland State University

Follow this and additional works at: https://pdxscholar.library.pdx.edu/open_access_etds

Part of the Psychology Commons

Let us know how access to this document benefits you.

\section{Recommended Citation}

Lord, Andreas D., "A Conditioning Model for the McCollough Effect" (1975). Dissertations and Theses.

Paper 2419.

https://doi.org/10.15760/etd.2416

This Thesis is brought to you for free and open access. It has been accepted for inclusion in Dissertations and Theses by an authorized administrator of PDXScholar. Please contact us if we can make this document more accessible: pdxscholar@pdx.edu. 
AN ABSTRACT OF THE THESIS OF Andreas D. Lord for the Master of Science in Psychology presented July 31, 1975

Title: A Conditioning Model for the McCollough Effect APPROVED BY MEMBERS OF THE THESIS COMMITTEE:

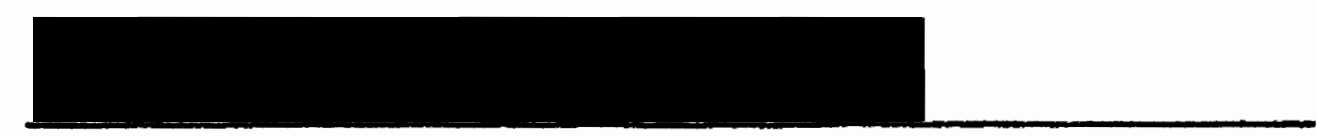
Gerald M. Murch, Chairman

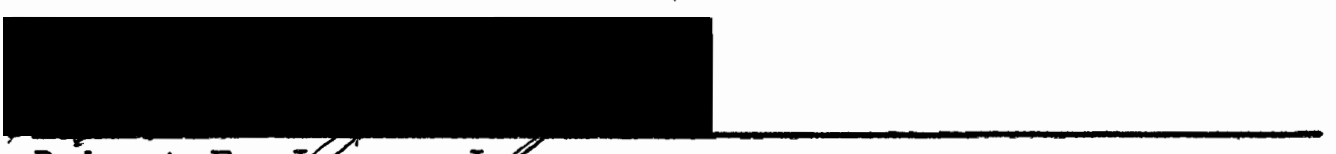
Robert E. Jones, Jr.

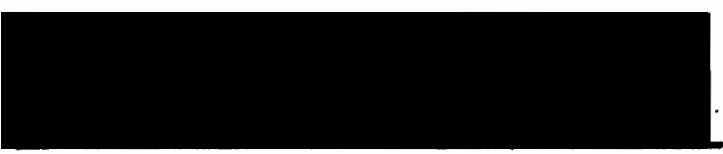

James Paulson

A model based on the laws of classical conditioning is posed as an explanation for the McCollough Effect, an orientation-specific color aftereffect. This model stands as an alternative to the color-coded edge detector hypothesis. Background and relevant issues are presented. Two experiments were performed. The first demonstrated that an auditory stimulus causes the effect to appear stronger to some subjects, a disinhibiting effect. It was also shown that some subjects experience spontaneous recovery of the effect after it has been extinguished. 
The second experiment demonstrated that the aftercolors will generalize to lines of varying orientation, including $45^{\circ}$. Subjects adapted to both red-vertical and green-horizontal lines saw mostly pink on test lines more than $30^{\circ}$ off horizontal. Subjects adapted to red only or green only saw the appropriate after-colors on patterns of all orientations between $0^{\circ}$ and $90^{\circ}$; These results conflict with the color-coded.edge detector theory and an explanaion in terms of classical conditioning is offered. 


\section{A CONDITIONING MODEL FOR THE MCCOLLOUGH EFFECT}

$$
\text { by }
$$

Andreas D. Lord

A thesis submitted in partial fulfillment of the requirements for the degree of

\section{MASTER OF SCIENCE \\ in \\ PSYCHOLOGY}

Portland State University

1975 
TO THE OFFICE OF GRADUATE STUDIES AND RESEARCH:

The members of the Committee approve the thesis of Andreas D. Lord presented July 31, 1975

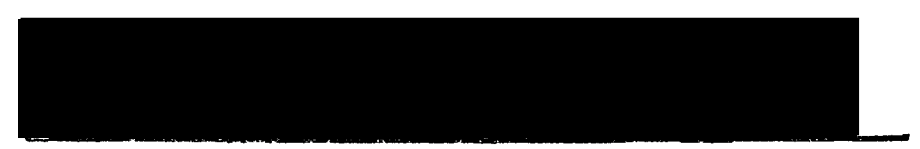

Gerald M. Murch, Chairman
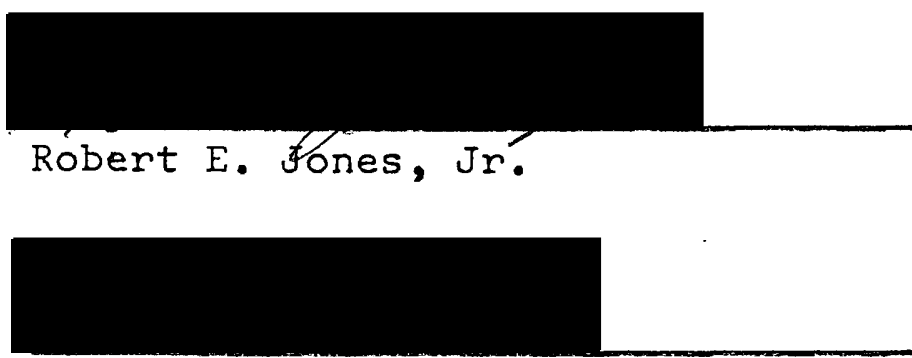

James Paulson

APPROVED :

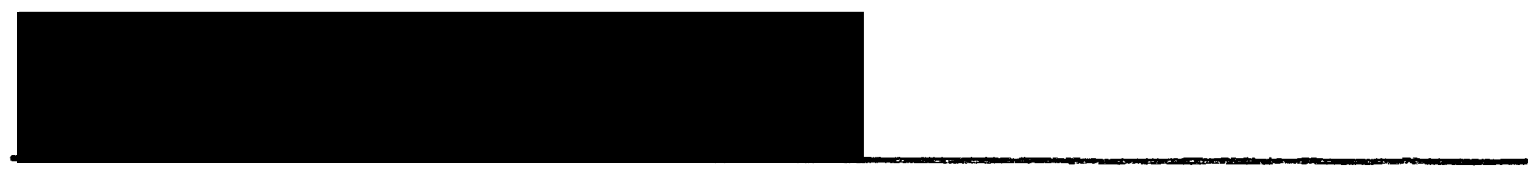

Ronald Smith, Head, Department of Psychology

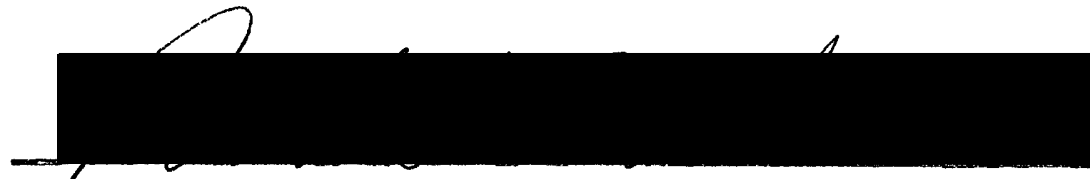

David T. Clark, Dean of Graduate Studies and Research

July 31,1975 


\section{ACKNOWLEDGMENTS}

I would like to thank Profs. Paulson and Jones for their support and advice in this endeavour. Professor Murch inspired this work of course, and I can't adequately thank him for his patience and help. He is a beacon on the stormy coastline of perception. Any praise this paper receives is his, any criticism mine. 
CHAPTER I -- BACKGROUND AND RELEVANT ISSUES ....... I

CHAPTER II - - THE CONDITIONING MODEL ............ 9

CHAPTER III -- EXPERIMENT I: PRESENCE OF CONDITIONING PHENOMENA

Method

External Inhibition

19

Disinhibition .........

Spontaneous Recovery

Discussion

CHAPTER IV -- EXPERIMENT II: STIMULUS GENERALIZATION

Method and Procedure.

Results and Discussion.

CHAPTER V -- SUMMARY AND IMPLICATIONS FOR FURTHER

REFERENCES 
CHAPTER I

BACKGROUND AND RELEVANT ISSUES

The McCollough effect is an orientation-specific aftereffect first discerned by McCollough (1965) as part of a series of experiments on gaze-contingent aftereffects. The various qualities (described below) exhibited by this and related effects suggest that they constitute an opportunity for the psychophysical investigation of color processing beyond the retinal level. In McCollough's original experiment, the subjects alternately inspected black vertical lines on an orange ground and horizontal lines on a blue field. After adaptation when presented with a colorless test'pattern of vertical lines on one half and horizontal lines on the other, subjects reported a blue-green aftereffect on the vertical field and an orange aftereffect on the horizontal field. Rotation of the test pattern $90^{\circ}$ caused the aftereffect to reverse (i.e., the horizontal lines, previously orange, became vertical and blue green; the vertical lines, now horizontal, became orange); rotation of $45^{\circ}$ eliminated the aftereffect. Physiological research by Hubel and Wiesel (1959, 1962) disclosed cell structures in the cortex of cats which were maximally sensitive to edges of different orientations. With this physiological evidence, McCollough argued that the effect was the result of the adaptation of vertical and horizontal edge detectors which had the added property of being sensitive to wavelength. Thus, in her experiment, the 
vertical edge detectors were adapted to orange, producing a blue-green aftereffect specific to vertical lines, and the horizontal detectors were adapted to blue, causing a horizontal specific orange aftereffect.

The color-coded edge detector hypothesis seemed adequate, if not formidable. Although the McCollough effect is superficially similar to the classical negative afterimage, which occurs retinally, there is ample evidence to dismiss any explanation of the effect in terms of afterimages (adaptation of retinal receptors). The McCollough effect requires neither fixation of a point on the inspection pattern nor an intense stimulus. It occurs after equal exposure to complementary colors, which would preclude the build-up of a negative afterimage. An afterimage dissipates after a few minutes, whereas the McCollough effect can last a day or longer. The McCollough effect has been shown to be orientation-specific, in that the effect weakens as the angular divergence of the red and green inspection patterns decreases. (Fidell, 1970). In addition, Murch (1969) showed that the McCollough effect does not follow Emmert's Law, which states that the size of the afterimage is directly proportional to the square of the distance of the test field. The McCollough effect covers the test field regardless of its size or distance from the observer. Color matching studies (Stromeyer, 1969) indicate 
that McCollough effect hues do not correspond to the expected hues of negative afterimages of the inspection colors.

From the available evidence, we may safely conclude that the color adaptation involved in the McCollough effect occurs post-retinally. An alternative to the color-coded edge detector hypothesis was first proposed by Harris and Gibson (1968). They discovered that lowering the contrast between the black and white lines of the test pattern weakens the effect, but that blurring the edges of the lines (while maintaining high contrast) does not impair the effect. They considered this evidence against the edge-detector hypathesis and felt called upon to conjure a new sensory unit, the "dipole." In somewhat nebulous terms, they assigned various properties to the dipole which would enable it to mediate the various characteristics of the McCollough effect. The invention of untestable physiological units to explain existing data is, quite fortunately, not yet an accepted procedure in psychophysics, and the dipole cannot be considered a valid alternative to the color-coded edge detector model.

The edge-detector hypothesis remained an attractive one. However, after McCollough's original work, a variety of similar field dependent color aftereffects were discovered. Hepler (1968) described a motion-contingent aftereffect. After inspection of horizontal red stripes moving up and similar green stripes moving down, subjects reported that white 
stripes appeared green when moving up and pink when moving down. Stromeyer and Mansfield (1970) illuminated one half of a spiral in green light, the other half in red. They alternated the direction of rotation every 10 seconds, at the same time alternating the colors. In white light, subjects reported aftereffects specific to the direction of rotation; when rotation was reversed, the aftereffect colors reversed. Spatial-frequency specific effects have been demonstrated (Breitmeyer and Cooper, 1972) by using similarly oriented red and green inspection patterns with different spatial frequencies. Appropriate aftereffect colors were reported specific to the frequehcies used in inspection. Riggs (1973) used inspection patterns of red and green, oppositely curved arcs, and found that aftereffects occurred specific to the direction of curvature. In addition to these field-contingent color aftereffects, other experiments have demonstrated color-contingent figural and motion aftereffects. Held and Shattuck (1971) showed that the tilt aftereffect (generated by inspection of oppositely tilted lines followed by a vertical test pattern) could be made contingent on the colors used during inspection. When oppositely rotating spirals are inspected in red and green light, the apparent motion of stationary:red:and green spirals is in the opposite direction of the inspection spirals. (Favreau, Emerson, and Corballis, 1972; Mayhew and Anstis, :1973). 
If the color-coded edge detector model is to be maintained, one is perforce led to hypothesize the existence of color-coded detectors specific to all the stimulus variables mentioned above, and this is exactly what occurred. Hubel and Wiesel's discovery of motion detectors in the primate cortex (1968) inspired the hypothesis that these, too, were color coded (Hepler). Color-codea cells specific to all the above field properties were proposed by various authors. Further research will undoubtedly reveal new contingent color aftereffects similar to the McCollough effect, which would have to be interpreted in similar terms. In fact, Wyatt (1974) has demonstrated aftereffects based on three stimulus dimensions rather than two. By using one inspection stimulus of red and green vertical lines differing in spatial frequency, followed by a similar inspection pattern of horizontal lines, he produced a McCollough-type aftereffect contingent on both frequency and orientation. He then demonstrated that adaptation to red and green verticals of different frequencies, followed by inspection of oppositely oriented colorless oblique lines of similarly different frequencies produced an aftereffect upon exposure to oppositely oriented oblique lines of equal frequency. The effect was a color aftereffect made contingent on a frequency aftereffect which was contingent on orientation. On the basis of this added dimension to previously established aftereffects, Wyatt suggested the existence 
of a population of orientation specific edge detectors coded for both wavelength and spatial frequency.

The color-coded edge detector hypothesis. clearly shows a tendency to become increasingly unwieldy as new effects are discerned, and as existing effects are made contingent on an increasing number of stimulus dimensions. In addition, Murch (1972) has shown that when the McCollough effect is produced in conjunction with a size aftereffect, (using the patterns developed by Blakemore and Sutton, 1969), the size aftereffect exhibits interocular transfer while the color effect does not. He interpreted this as evidence that the same neural units could not be responsible for both aspects। of the effect. In reply, Coltheart (1973) pointed out that a small but evident loss of strength of the size aftereffect in the contralateral eye suggest the possibility of two. populations of detectors, one monocularly driven, one binocularly driven, the former color adaptive and the latter not. The same criticism could be levelled at Mayhew and Anstis (1972), who obtained results similar to Murch's with respect to a color contingent motion aftereffect (i.e., the motion aftereffect, made contingent on color, failed to show binocular transfer). Further experimentation (Over, Long, and Lovegrove, 1973), with monoptic and dichoptic presentation of contour and color stimuli supports the hypothesis that binocular spatial detectors are not tuned to wavelength, and 
an explanation in terms of monocular color-coded detectors was offered.

In a later experiment controlling for this possibility, Murch (1974) had subjects inspect a colorless alternately rotating spiral through one eye, while alternating red and green stimulation to the other eye. If monocular colorcoded motion detectors were responsible for the effect, the experimental condition should produce no effect in either eye. If an association between monocular color detectors and binocular motion detectors underlie the effect (as Murch suggested), then an effect should be produced in the color adapted eye, but not in the motion stimulated eye. The results of this experiment showed the latter to be the case.

The new physiological evidence provided by Hubel and Weisel (1968) has been cited in support of both an associational model (Murch and Hirsch, 1972; Murch 1972) and the monocular color-coded edge detector hypothesis (Lovegrove and Over, 1973; Over;:Long and Lovegrove, 1973). Murch and Hirsch theorize that the adaptation of monocular opponent-process color detectors, which Hubel and Wiesel found in the primate lateral. geniculate nucleus, occurs in conjunction with the stimulation of cortical edge detectors, which Hubel and Wiesel found to have little sensitivity to wavelength. They buttressed this argument experimentally by showing that the presentation of 
an unlined color stimulus before typical McCollough adaptation caused a shift in the hue of the aftereffect. This would not be expected if the adaptation of color-coded edge detectors was responsible for the effect (Hirsch and Murch, 1972). Coltheart (1973) analyzed the results of the physiological research in a way which suggests a hierarchical pattern of sensory attributes. He states that as one progresses up the visual system, color specificity diminishes, orientation specificity increases, and the monocularity of the sensory units decreases as binocularity increases. He further suggests that the

association between monocularity and colorspecificity [in the McCollough effect] can be seen as a natural. . consequence of the fact that there exists a stage in the visual system that is a) low enough. - to retain some degree of color specificity, yet b) high enough to possess orientation specificity, though c) not high enough for binocularity to have replaced monocularity.

It is at this stage, presumably, where the monocular colorcoded edge detectors lie. 
THE CONDITIONING MODEL

An alternative explanation of the mechanism underlying the McCollough and related effects has been mentioned by Hepler (1968) and Mayhew and Anstis (1972), and has been more fully elaborated by Murch (1975). This explanation considers the McCollough effect to be the result of simultaneous classical conditioning of the visual system. In the context of this model, the McCollough inspection patterns are considered to pair an unconditioned stimulus (color) with a conditioned stimulus (specifically oriented lines or edges). After a suitable number of pairings, the unconditioned response (the adaptive response of the visual system to color).is-conditioned to the presentation of the conditioned stimulus (the test lines).

Many of the data generated by previous experimentation on the McCollough and related effects fits readily into the context of the conditioning model. Several experimenters have observed the desaturation of the aftereffect as the test pattern deviates from the inspection pattern. Thus, Stromeyer (1972) found that the strength of the McCollough effect diminished as the frequency of the test pattern deviated in either direction from that of the inspection patterns. Teft and Clark (1968) found that as the difference between line densities of the inspection and test patterns increased, the 
strength of the aftereffect decreased. McCollough and Gerrein (as cited by Hirsch, 1971) used red and green vertical and diagonal inspection patterns to produce an effect on vertical and horizontal test patterns which differed from the effect produced on test patterns identical to the inspection patterns. Similar results have been obtained when field effects are made color contingent. Lovegrove, Over, and Broerse (1972) reported that the color contingent motion aftereffect is greatest when the test colors are identical to the inspection colors, and is diminished when the test colors are the complements of the inspection colors.

The dominant pattern of such results permits one to draw the conclusion that the greater the similarity between the inspection patterns and the test patterns, the stronger is the resulting aftereffect (with one exception, discussed below).

In keeping with the edge-detector model, the above authors have advanced explanations in terms of overlapping adaptation of detectors responding maximally to the specific characteristics of the stimulus attribute upon which the aftereffect is made contingent. The conditioning model offers a compelling explanation in terms of stimulus generalization. The basic characteristic of stimulus generalization is the elicitation of the conditioned response by a range of stimuli other than the one to which the response is conditioned. The strength of the elicited response is inversely related to 
the difference between the generalized stimulus and the conditioned stimulus. The data relative to degree of inspectiontest similarly fit into this model easily, with the notable exception of Riggs' color aftereffect contingent on curvature (1973). He found that the strongest effect occurred with the test pattern showing the greatest curvature, not the test pattern identical to the inspection pattern. Stromeyer (1974) explained this exception by suggesting that mutually cancelling effects are produced over distributions of edge-detectors stimulated through each hemi-retina by red and green lines of positive and negative slope. This exception is more easily explicable in the context of the conditioning model if one considers that the effect is being conditioned to a specific attribute of the inspection pattern, (the CS) and not to the inspection pattern itself. In this case, the aftereffect can be interpreted to be conditioned to degree of curvature, rather than to the specific curvature of the inspection lines. If this is so, one would expect the strength of the aftereffect to increase as the radii of curvature in the test pattern decreases, and to diminish as the radius of curvature increases. Riggs obtained exactly these results. Applying a similar analysis to the McCollough effect leads to the hypothesis that the primary attributes of the inspection patterns to which the response is conditioned are degree of vertical orientation and 
horizontal orientation. The strength of the conditioned response will be a direct function of the degree to which the test patterns possess these attributes. Since the inspection patterns are already optimally vertical and horizontal, the strength of the aftereffect can only diminish as the test patterns deviate from the inspection patterns.

Fidell (1970) showed that as the angular divergence between the lines of the red and green inspection patterns decreased; the strength of the aftereffect decreased, until it broke down at 11 degrees divergence. She interpreted this as the result of the adaptation of heavily overlapping populations of edge-detectors to both red and green; such simultaneous adaptation would produce little or no aftereffect. An explanation in the context of the conditioning model would be similar, except that it would not necessitate the assumption of color coded edge-detectors. If one considers that the visual system fails to discriminate between the two conditioned stimuli, the unconditioned response to both red and green is being conditioned to phenomenally identical stimuli. When these stimuli are presented in the test pattern, both responses occur to both stimuli, precluding the manifestation of an aftereffect.

Most of the data relevant to the strength of the McCollough effect can be explained in terms of stimulus generalization or discrimination (or failure to discriminate). 
13

The present experimentation has two main purposes. The first is to determine whether the MaCollough effect exhibits other characteristics common to classically conditioned responses, specifically disinhibition, external inhibition, and spontaneous recovery. The second is to attempt to generate a stimulus generalization gradient for the McCollough effect, and to note what effect, if any, changes in inspection might have on such a gradient. 
EXPERIMENT I: PRESENCE OF CONDITIONING PHENOMENA

According to the conditioning model, repeated presentation of the CS (vertical and horizontal lines) in the absence of the UCS (color) should extinguish the CR (MCCollough effect). Previous research (Skowbo, Gentry, Timney and Morant, 1974) has shown that the effect does indeed dissipate far more rapidly when subjects are stimulated by colorless grids after adaptation, as opposed to other types of visual stimuli (including normal visual activity). The first group of subjects in this experiment underwent repeated exposure to the CS until the effect was extinguished; this group will henceforth be referred to as the extinction group.

The second group of subjects underwent the same conditioning and extinction process as the extinction group. The only difference was that an auditory stimulus was presented to each subject simultaneously with the CS on specific trials during the extinction process. Thus, extinction curves for the two groups could be compared for differences in response strength, indicating the presence or absence of the inhibitory phenomena. This group will be referred to as the external stimulus group.

External inhibition is defined as the temporary decrease in the strength of a conditioned response caused by the presentation of an extraneous stimulus on an acquisition trial. 
With the McCollough effect, the $C R$ can only be measured in the absence of the UCS (color). Thus, all test trials are by definition extinction trials. By presenting an auditory stimulus on the first test trial it was hoped to discern whether the stimulus had a different effect on response strength when the $C R$ was at its theoretical maximum than. when the $C R$ had diminished somewhat. Since the extraneous stimulus was presented on the first trial, the conditioning model would predict little or no inhibitory effect. The model would also predict that the stimulus should cause the $C R$ to be disinhibited when presented on a later exhibition trial. Disinhibition is the increase in response strength during extinction caused by the presentation of an extraneous stimulus. For the second group of subjects, the external stimulus group, each subject received an auditory stimulus on the first extinction trial and on a later trial after response strength had diminished to a specific level.

Some experimenters have informally observed that after the McCollough effect has been: dissipated somewhat by repeated presentations of the achromatic test patterns, a period of normal visual stimulation, followed by another presentation of the test pattern, causes the effect to appear stronger than when testing was originally discontinued. Mckay and McKay (1974) observed such an increase in the strength of the effect after sleep. The color-edge detector hypothesis offers little in the way of an 
explanation for such recovery. The conditioning model can account for this phenomenon in terms of spontaneous recovery of the conditioned response. Spontaneous recovery is normally observed after a period of time has elapsed between extinction of the $C R$ and another presentation of the CS.

Method

Subjects were male and female psychology students who reported obtaining the McCollough effect during a screening session. At least eight days separated screening and experimentation. Fourteen subjects were run in each group, seven in each test condition. Subjects were seated at a distance of 3.1 meters from the viewing screen, with their heads in a chin rest. Patterns were projected onto the screen at a visual angle of $8^{\circ}$ by three Kodak Carousel projectors with the filters (Wratten $34 \mathrm{~A}, 56$, and $\mathrm{ND} .70$ ) mounted in front of the barrels. All stimulus slides were made with photographic black and colorless slide positives, all of equal spatial frequency ( $1.9 \mathrm{cyc} / \mathrm{deg})$. The projected inspection and test patterns all described angles of $11.5^{\circ}$ vertically and $11.5^{\circ}$ horizontally. External stimulus subjects were administered a pure tone $(700 \mathrm{cps})$ to both ears on the appropriate trials through earphones connected to a reelto-reel tape player. 
Procedure

Adaptation for the extinction and inhibition groups consisted of ten second exposure to vertical magenta and horizontal green inspection patterns (Wratten filters $34 \mathrm{~A}$ and 56) alternating with an interstimulus interval of 20 seconds, for 12 alternations. Testing continued until subjects reached an extinction criterion of three consecutive "no color" trials; if a subject failed to reach criterion after 50 trials, the procedure was terminated.

Response strength was ascertained on each test trial by the method of direct magnitude estimation. Marks (1974) has asserted that reliable data on subjective differences between sensory experiences can be achieved by having the subject assign an arbitrary number to represent the strength of the initial sensation, and assign numbers to succeeding sensations relative to the first. Stromeyer (1969) used this method in an experiment on the effect of variations in inspection color on the strength of the aftereffect, and obtained satisfactory results. In this case, subjects were asked to assign a number to represent the strength of the initial aftereffect, and to assign numbers to succeeding effects relative to the first, letting zero represent no color. They were also asked to name the color of the effect and to mention changes in color should they occur. The responses of each subject could then be adjusted to fit a 
scale of response strength from zero (representing no color) to one (representing the maximum response strength reported by each subject). Subjects in the extinction procedure were tested to criterion, as described above. Subjects in the external stimulus procedure were similarly tested, except that they received an auditory stimulus on the first test trial, and on the trial immediately following the trial on which they first reported a response strength less than or equal to $33 \%$ of their previously reported maximum response strength. The stimulus was presented simultaneously with the test pattern and was maintained until the test pattern went off. All subjects in both procedures wore earphones.

To test for spontaneous recovery, subjects in both the extinction and disinhibition groups were asked to return at least one hour after the extinction of the effect. They were tested with one 10 second presentation of the test slide, and asked to make a direct magnitude estimation of any observable effect, using the same scale which they had used before.

Results

Two subjects in the extinction group, and one in the external stimulus group, failed to report a consistent weakening of the effect over 50 trials, and one failed to give the appropriate color response to the horizontal test pattern. Four subjects in the external stimulus group failed to reach $33 \%$ of maximum response strength over 50 trials, and one failed to observe an effect on the first three test trials. 
These subjects are not included in the data. Thus, the data reflect results obtained from 11 subjects in the extinction group, five in the horizontal test condition and six in the vertical, and eight subjects in the inhibition group, four in each test condition. In the extinction group, trials to criterion ranged from six to 38 in the horizontal test, and nine to 42 in the vertical. In the external stimulus group trials to criterion ranged from 10 to 38 . Three external stimulus subjects failed to reach criterion in 50 trials, but had reached response strength levels of $.25, .10$, and .20 , showing a consistent dissipation of the effect, and were included in the data.

\section{External Inhibition}

Mean response strengths for the first three test trials were computed for each group and appear in Fig. 1. Five of the 11 extinction subjects, and four of the eight external stimulus subjects, reported maximum responses on the first trial. The difference between mean response strengths of the two groups on the first trial proved to be insignificant $(t=.305)$ at the $5 \%$ level. The extraneous stimulus apparently had no discernible effect when presented on the first trial, although one subject reported no color on the first (inhibition) trial, and an adjusted strength of .25 on the next trial. The difference in mean response strengths on the first trial is an effect of this subject. 

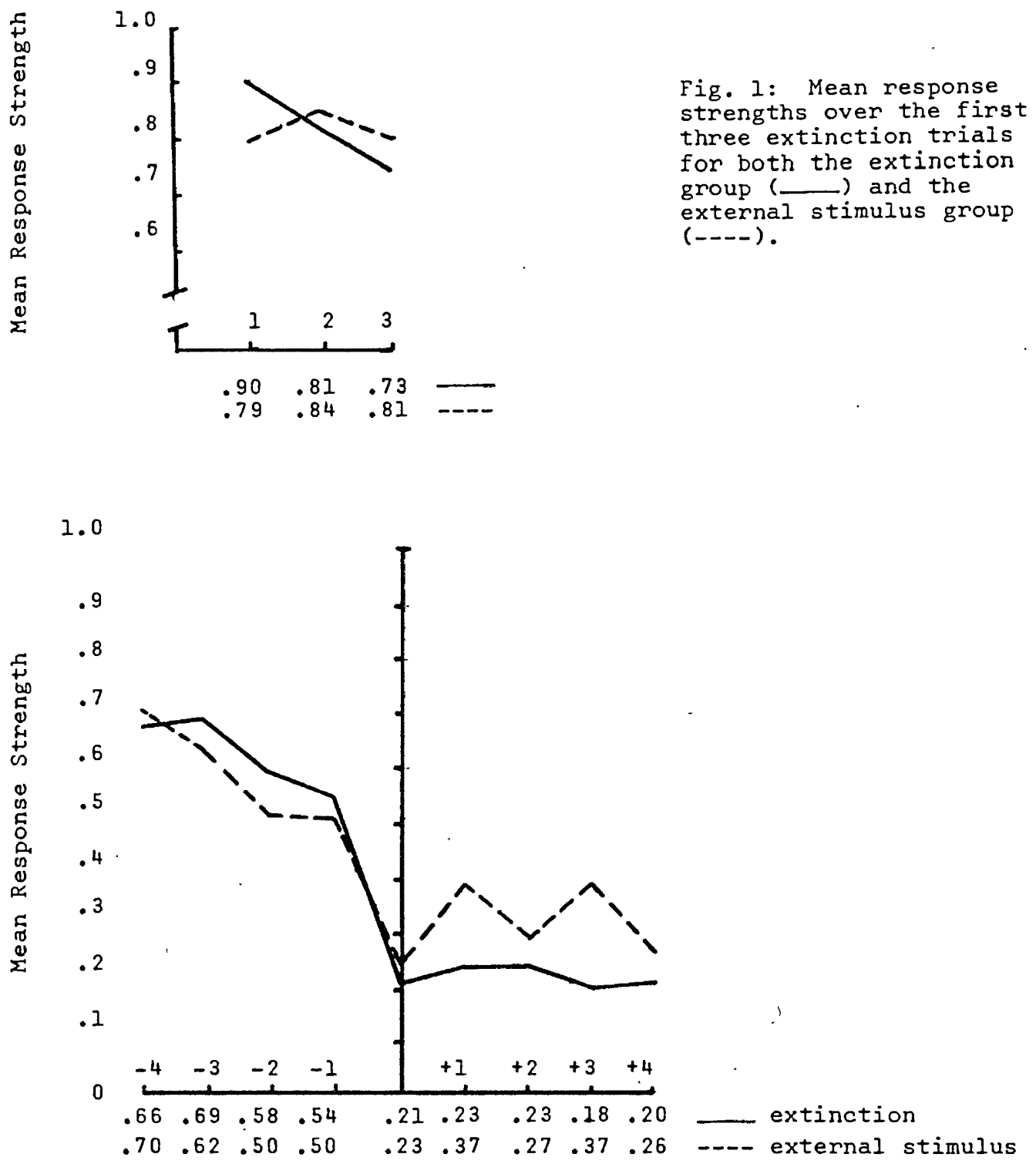

Fig. 2: Mean response strengths for both groups for the four trials before and after the zero trial. Disinhibition subjects received the auditory stimulus on trial +1 . 
Disinhibition

The auditory stimulus was also presented to external stimulus subjects on the trial immediately following the trial on which the subject had first given a response strength estimate of $33 \%$ or less of his previously reported maximum response strength: As one might expect, the disinhibition trial fell on different trial numbers from one subject to the next, specifically on trials $4,8,9,9,14,20,25$ and 28. In order to summarize the data, mean response strength was calcualted for the 5 trials preceding the tone, the tone trial, and the three trials following the tone.

To compare these results with the extinction group, the trial preceding the tone was designated as trial 0 , the one previous to it as $-I$, and the one following it as +1 (the latter of course being the disinhibition trial). The defining attribute of trial 0 was that it was the first trial

for each subject in both groups on which response strength reached $33 \%$ (or less) of maximum. Mean response strengths could then be computed on trials -4 to +4 for each extinction subject, and be compared with those obtained for the inhibition group. Trial +1 fell on trials number $5,5,7,7,8,10,12$, 15, 20, 26 and 34 for the extinction group. The two extinction curves are presented in fig. 2, with a summary of mean response strengths over the nine trials for both groups. It did 
occur in some cases that a subject did not give a response on one or more of the trials -4 to +4 : This would be the calse if the zero trial occurred before the fifth extinction trial. Three subjects in the extinction group reported $33 \%$ or less response strength on the fourth extinction trial; thus, they are not included in the data for trial -4 , since they experienced only three trials before the zero trial. One subject in the external stimulus group reached the zero trial on the third extinction trial, and is thus not included in the data for trials -3 and -4 . Similarly, some subjects reached extinction criterion before trial +4. Again, if a subject did not experience a trial, the mean response strength was calculated only for the remaining subjects. This accounts for the flattening of the two extinction curves in Fig. 2. In the external stimulus group, one subject reached criterion on trial +2 ; thus the data for trials +3 and +4 are the means of the remaining seven subjects. In the extinction group, one: subject reached criterion on trial +2 , and another on trial +3; having reached criterion, they were not included in the data for later trials.

Two of the eleven subjects in the extinction group reported an increase in response strength from trial 0 to trial 1. One subject went from a no color response to .90 , the other from .17 to .25 . Four others reported a decline in response strength. The other five reported no change. In 
the external stimulus group, three of eight subjects recorded a rise in response strength; from .25 to $.50, .33$ to .67 , and no color to .75 . Only one subject reported a decline from trial 0 to trial 1. The other four reported no change. The differences in mean response strength between groups, as shown in Fig. 2, are significant on trials +1 and +3 $\left(t_{1}=4.5, t_{3}=2.78, p=.05\right)$. The difference in mean response strength between trial 0 and trial 1 in the external stimulus group is significant $(t=2.02)$ at the .10 level

\section{Spontaneous Recovery}

of 14 subjects who volunteered to return an hour after extinction of the effect, four experienced partial recovery of the effect, with adjusted magnitude estimations of $.10, .20$, .50 , and .75 .

\section{Discussion}

The results seem to indicate that some disinhibition of the McCollough effect occurred in the external stimulus group. Two of the three subjects who reported a stronger effect on trial +1 commented that the "tone really brought out the yellow" and that "the color got stronger" on the experimental trial. Such subjective comments may. be of greater importance than the statistical data. Subjects were being asked to make rather fine judgments of a color effect on a test pattern of 
uniform orientation without being able to contrast the test pattern with a neutral stimulus. Also, the variance in the number of trials to criterion between subjects makes a meaningful comparison between groups on specific trials more difficult. This is why an interval of oniy nine trials was chosen to compare the two groups. Still, it seems clear that disinhibition of the effect occurred in at least two of the eight experimental subjects (the other subject who reported an increase from trial 0 to trial 1 showed a rather unstable extinction curve); this result is inconsistent with the colorcoded edge detector hypothesis, whereas the conditioning model would predict such a disinhibiting effect. Further, the fact that the tone had no effect on initial response strength, yet influenced response strength on the later trial, would also be predicted by the conditioning model. This is because the extraneous stimulus should cause an inhibition of response only during acquisition. Since response strength is at its theoretical maximum on the first extinction trial, no disinhibiting effect would be expected, and since it is not an acquisition trial, no inhibiting effect would be expected. The fact that the tone had no effect on the first trial, yet had a disinhibiting effect (for some subjects) on a later trial, is entirely consistent with the conditioning paradigm. The edge detector hypothesis would not seem to have an explanation for such results, nor a basis for predicting them. 
Four subjects reported spontaneous recovery of the effect after it had been experimentally extinguished. It should be emphasized that this recovery occurred after the effect had been extinguished to criterion and is wholly at odds with the color-edge detector hypothesis. If color-edge detectors underlie the effect, then extinction of the effect occurs when those cells adapted by the inspection patterns regain full strength. Once this has occurred, further observations of the effect could not occur without further adaptation. It should also be mentioned that spontaneous recovery normally occurs after an interval during which the subject is not presented with the CS.' 'In this experiment, subjects engaged in normal visual activity, which would include some horizontal and vertical stimulation. One might expect slightly greater recovery of the effect if subjects were visually deprived between extinction and the recovery trial. In their experiment on decay rate of the McCollough effect, Skowbo et. al. (1974) found that the effect dissipated more quickly after 10 minutes of natural stimulation than after 10 minutes of darkness (although degrees of decay were approximately the same after 50 minutes). 
CHAPTER IV

EXPERIMENT II: STIMULUS GENERALIZATION

The strength of the McCollough effect has been found to decrease as the orientation of the test lines deviates from the orientation of the inspection lines. The purpose of this experiment was to establish a generalization gradient for the McCollough effect from which predicitons might be made concerning the effects of changes in inspection conditions on the strength of generalized responses.

Method and Procedure

Subjects were 16 graduate and undergraduate students screened as before. Eight were adapted in the manner described above, except that they viewed the red and green. inspection patterns for 20 alternations instead of 12 . The added adaptation would serve to help maintain response strength at a stable level throughout the testing procedure, preventing decay of the effect from affecting magnitude estimations. Four additional subjects were adapted only to red vertical patterns, 20 presentations of 10 seconds with an inter-stimulus interval of 50 seconds. The remaining four subjects were similarly adapted only to green horizontals. 
Test patterns were 11 black and colorless patterns with lines oriented horizontally, 10 degrees off horizontal, 20 degrees off horizontal, etc., to vertical, plus one pattern at 45 degrees. Each test pattern was presented once to each subject for 10 seconds, with an ISI of 20 seconds. Subjects were asked to name whatever color they saw on each pattern, and to make a numerical direct magnitude estimation of the strength of the effect. To further mitigate the effects of aftereffect decay, four random sequences of presentation were generated. Using the data from the extinction subjects in the previous experiment, mean response strengths over the first eleven trials were obtained. Using these mean response strengths, the four test presentation sequences were designed so that the average response strength of all test stimuli over all four sequences was the same. Thus, if each subject in a group of four views a different sequence of test stimuli, any difference in mean response strength between test stimuli should not be due to decay of the effect. Each sequence was used twice in the red-green inspection group, once each in the redonly and green-only groups.

Results and Discussion

Mean adjusted response strengths for each of the eleven test patterns are given in fig. 3 for the red-green group. Color names appear in the appendix. Surprisingly, mean response strengths do not differ significantly as a function 


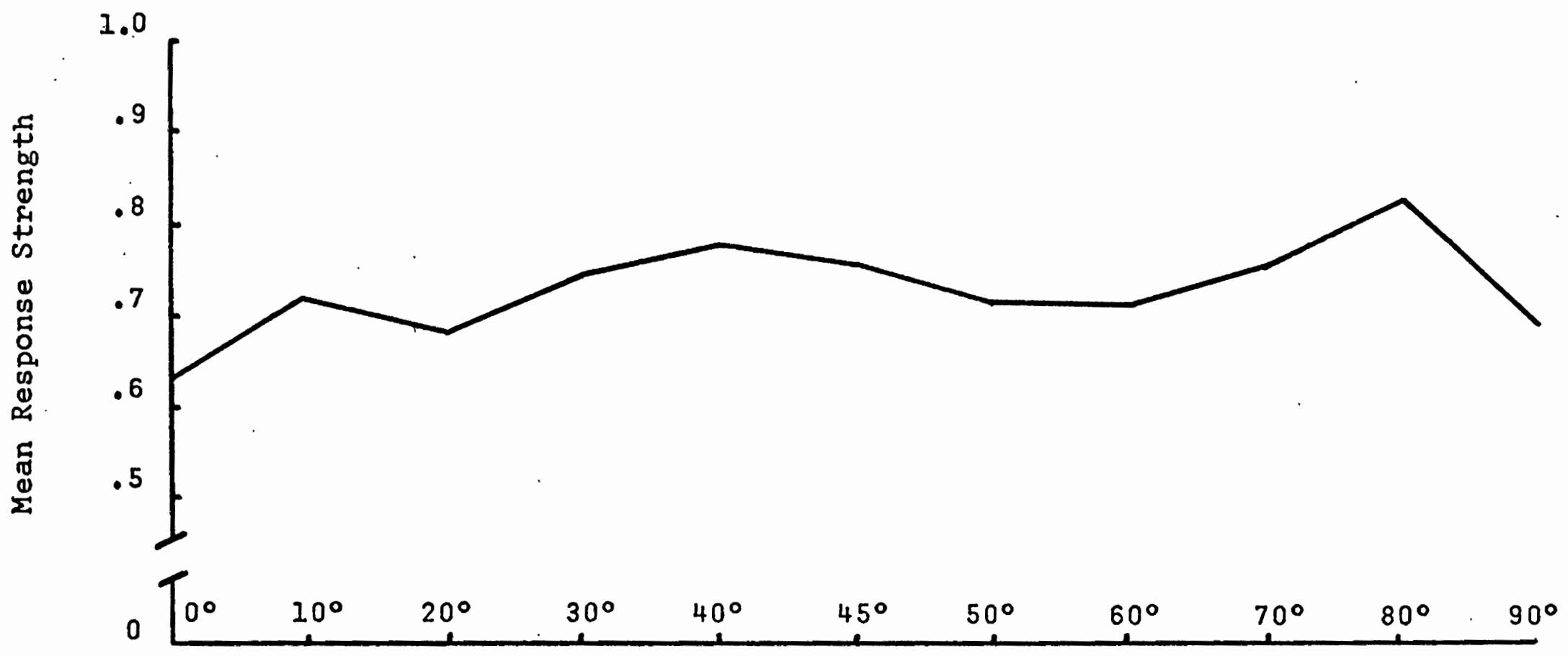

Fig. 3: Mean response strength over all test orientations (in degrees off horizontal) for subjects adapted to both inspection patterns. Color names appear in the appendix. 
of test orientation. Such results are not consistent with previous experimentation and should be approached with some caution. However, it would seem unlikely that the sixteen subjects in this experiment were all unreliable in reporting the presence of an effect when there was none. With the test orientation at $45^{\circ}$, the edge-detector hypothesis would predict the absence of any color. This would be because the oblique lines fail to stimulate the edge-detector populations which were adapted during inspection. The conditioning model offers two possible explanations for the absence of color at $45^{\circ}$. First, the color responses conditioned to the vertical and horizontal lines in inspection have failed to generalize to the test stimulus. Second, the color responses have in fact generalized to the test stimulus, but cancel each other. If the second were the case, and if one response were more strongly conditioned during inspection than the other, one would expect to observe that response on the oblique test pattern. A glance at the color names associated with the various test patterns shows that the appropriate response to the red vertical inspection pattern (green, greenish-yellow; yellow) seems to have generalized over a greater number of test orientations than the appropriate green-horizontal response (pink, red, orange). This is explicable if one imagines that the red-vertical response was more strongly conditioned during inspection. This may in fact have been the case. Of the 10 subjects who failed to reach extinction 
criterion in the first experiment, six were tested with the vertical pattern, four with the horizontal pattern. Only one of the former experienced a significant weakening of the effect, whereas two of the latter did. Also, mean trials to criterion for the rest of the subjects in the first experiment was 20 for those tested with the horizontal pattern, 27 for those tested with the vertical pattern. Apparently, the red vertical stimulus may have conditioned a somewhat stronger response than did the green horizontal. If this were so, it would account for the predominance of red-vertical-appropriate color responses in the generalization procedure. It would also permit the prediction that the generalization curve for subjects adapted only to green horizontals would be steeper than that for subjects adapted only to red verticals. Generalization gradients for subjects in the single. color-orientation groups appear in Fig. 4. Color names appear in the appendix. The predicted effect apparently. did not occur, since mean response strength differences between the two conditions do not vary when one compares the results of patterns that are of equal degrees off vertical and horizontal. It may be that the green-horizontal response generalizes less well, when a competing response is being conditioned.

The most remarkable aspect of these results is that the McCollough colors were clearly seen on:test patterns up to $90^{\circ}$ deviant from the inspection patterns. Of 88 test 
-w Green Horizontal Inspection

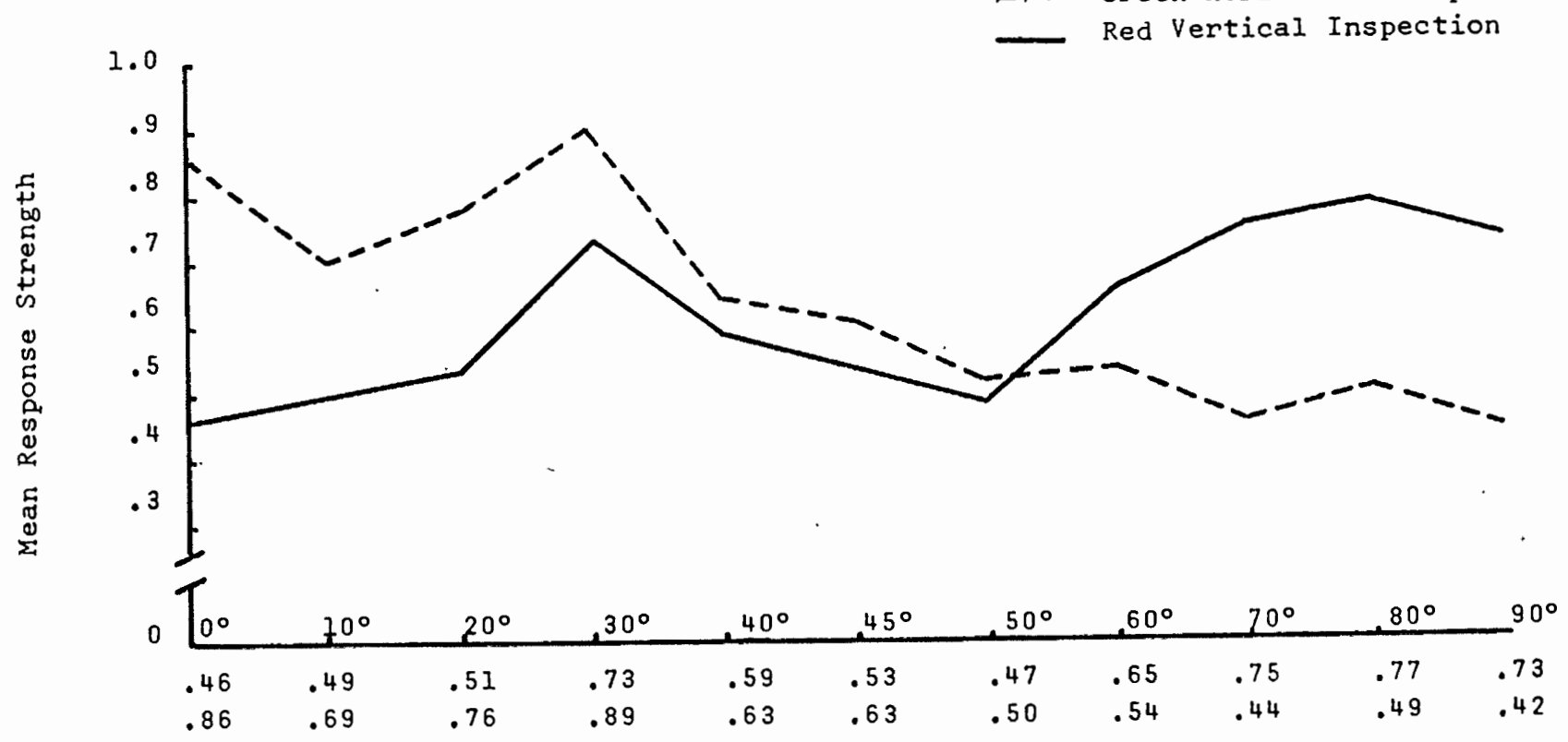

Fig. 4: Mean response strength over all test orientations (in degrees of horizontal) for subjects adapted to only red-vertical or green-horizontal. Color names appear in appendix. 
presentations in this procedure, only two "no color" responses were given. Such a result is entirely inconsistent with the . color-coded edge detector hypothesis, which would predict no color effects on test patterns deviating more than 45 degrees from the inspection pattern. The McCollough inspection patterns evidently condition responses to a wide range of line orientations, well beyond $45^{\circ}$ deviation from the inspection lines.

Previous experimentation (e.g., Fidell, 1970) measured the strength of the effect by having subjects add an appropriate amount of the original adapting hue to the test stimulus until the effect was neutralized. This technique rests on the assumption that subjects see only varying strengths of the same color effect as the test lines change in orientation to $45^{\circ}$. The results of this experiment seem to indicate that the effect does not desaturate as test-line orientation changes, but that the color of the effect actually changes. If the hue-addition technique were employed, and if the deviating test lines were actually eliciting an effect containing more of the hue being added, the subject would add progressively less of the hue necessary to neutralize the effect. Red-vertical and green-horizontal inspection is thus seen to produce an effect on an oblique test pattern which is some combination of the two appropriate aftereffect colors, rather than merely one desaturated aftereffect color. It is true that the appropriate aftereffect colors should appear 
strongest on the $0^{\circ}$ and $90^{\circ}$ test patterns. However, in this experiment, subjects were asked to make magnitude estimations of the strength of the effect irrespective of color. Previous methods have assumed that, for instance, a lesser degree of pink seen on a test pattern $30^{\circ}$ off horizontal indicated a weaker effect. The present results indicate that the effect at $30^{\circ}$ does not necessarily have less pink, but more green, and that it is the color of the effect, and not its strength, that changes as the test lines deviate from vertical and horizontal. The fact that subjects adapted to inspection lines of one color and orientation reported the appropriate color effect on test lines oriented well beyond $45^{\circ}$ off inspection orientation suggests that the McCollough effect hues result from varying combinations of both after-colors. This is a possible explanation for the fact that the McCollough colors do not correspond to the complements or expected negative after-images of the inspection colors (Stromeyer, 1969). The present results allow for the possibility that, e.g., the aftereffect elicited by a horizontal test pattern (after red-vertical, greenhorizontal inspection) is not strictly a response to one inspection pattern, but the combined responses to both inspection patterns. In the context of the conditioning model, one can consider that each test stimulus acts as a CS (or generalized $(S)$ to both $C R$ 's. 
CHAPTER V

SUMMARY AND IMPLICATIONS FOR FURTHER RESEARCH

An auditory stimulus caused at least two of eight subjects to experience an increase in the strength of the McCollough effect. Four of 14 subjects reported a partial recovery of the effect some time after it had been extinguished. The McCollough effect was found to generalize to a broad range of line orientations differing from those used in inspection. All these results are inconsistent with a color-coded edge detector hypothesis, but could be readily incorporated into a conditioning model.

Recent experimentation (UhIarik and 0sgood, 1974) has shown that changes in the spatial characteristics (width) of the black lines of the inspection patterns have a greater effect on response strength than similar changes in the colored lines. Predictably, this was explained by suggesting that some color-coded edge detectors had the added property of being sensitive to width. Further research should concentrate on defining more explicitly the relationship between the conditioned stimuli and the conditioned responses, generating more reliable extinction data, and investigating other classical conditioning phenomena (reconditioning, discrimination, compound conditioning), as they apply to the McCollough effect. A replication of these experiments using 
a colorimeter to obtain more accurate measures of response strength would be beneficial. While the method of direct magnitude estimation is sufficient to establish general trends, and to compare effects clearly differing in strength (as in the disinhibition series), fine discriminations are difficult.

The generalization experiment suggest a new approach to the study of the changes in the effect caused by deviations in test line orientation. Further experimentation should also use more sophisticated methods to discern the hue and stpength of the effect. The evidence that the color of the effect changes as the orientation of the test lines change suggests experimental research into the nature of this change, including differences in aftereffect hue on similar test stimuli caused by changes in inspection colors. The McCollough effect continues to offer a unique opportunity for the psychol-physical investigation of visual functioning beyond the retinal level. 
REFERENCES

Blakemore, C. and Sutton P., Size adaptation: a new aftereffect, Science, 1969, 166; pp. 245-247.

Breitmeyer, B. G. and Cooper, L. A., Frequency specific color adaptation in the human visual system, Perception and Psychophysics,. 1972, 11, pp. 95-96.

Coltheart, M. Color specificity and monocularity in the visual cortex, Vision Research, 13, 2595-2598, 1973.

Favreau, O. E., Emerson, V. F. and Corballis, M. C., Motion perception: a color contingent aftereffect, Science, 1972, 176, pp. 78-79.

Fidell, L. Orientation specificity in chromatic adaptation of human "edge detectors;" Perception and Psychophysics 1970,8 , pp. 235-237.

Gibson, A. and Harris, C. The McCollough Effect: color adaptation of edge detectors or negative afterimages? Paper presented at the meeting of the Eastern Psychological Association, 1968.

Harris, C. Effect of viewing distance on a color aftereffect specific to spatial frequency, Psychonomic Science, 1970, 21, 350 (abstract).

Harris, C. and Gibson, A. Is orientation-specific color adaptation in human vision due to edge-detectors, afterimages, or "dipoles"? Science, 1968, 162, pp. 1506-1507.

Held, R. and Shattuck, S. R., Color and edge-sensitive channels. in the human visual system, Science, 1971, 174 , Pp. 314-316.

Hepler, N. Color: a motion-contingent aftereffect, Science $1968,162, \mathrm{pp} .376-377$.

Hilgard, E. R., and Marquis, D. G., Conditioning and Learning, rev. by Kimble, Appleton Century.Crafts, 1961.

Hirsch, J. Variation in a Color-line Aftereffect Due to Color Adaptation, Masters Thesis, Portland State University, 197I. 
Hirsch, J. and Murch, G. Variation in hue of a contourcontingent aftereffect due to color adaptation during inspection of the stimulus patterns, Perception and Psychophysics, 1972, 11, Pp. 406-409.

Hubel, D. H. and Weisel, T. N. Receptive fields of single neurones in the cat's striate cortex, Journal of Physiology, 1959, 148, PP. 574-591.

Hubel, D. H. and Weisel, T. N. Receptive fields, binocular interaction and functional architecture in the cat's visual cortex, Journal of Physiology, 1962, 160, pp. 106-154.

Hubel, D. H. and Wiesel T. N. Receptive fields and functional architecture of monkey striate cortex, Journal of Physiology, 1968, 195, pp. 215-263.

Lovegrove, $W_{\text {. }}$ and Over, $R$. Color Selectivity in orientation masking and aftereffect, Vision Research, 1973, 13, :Pp. $895-902$.

Lovegrove, $W_{.}$, Over, $R_{\text {. and Broerse, }}$., Color selectivity in motion aftereffect, Nature, 1972, 236, pp. 334-335.

Mackay, D. and Mackay, V. What causes decay of patterncontingent chromatic aftereffects? Vision Research, 1974,14 , pp. 1285-1288.

Marks, L. E. Sensory Process: the new psychophysics, Academic Press, 1974.

Mayhew, J. E. W. and Anstis, S. M. Movement aftereffects contingent on colour intensity and pattern, Perception and Psychophysics, 1972, 12, pp. 77-85.

McCollough, C. Color adaptation of edge detectors in the human visual system, Science, 1965, 149, pp. 1115-1116.

Motokawa, K. Physiology of Color and Pattern Vision, Igaku Shoin, Tokyo, 1970.

Murch, G. M. Size judgments of McCollough afterimages, Journal of Experimental Psychology, 1969, 81, pp. 44-48.

Murch, G. M. Binocular relationships in a size and color orientation specific aftereffect, Journal of Experimental Psychology, 1972, 93, PP. 30-34.

Murch, G. M. Color contingent motion aftereffects: single or multiple levels of processing, Vision Research, 1974, 14 , pp. $1181-1184$. 
Murch, G. M. and Hirsch, J. The McCollough effect created by complementary afterimages, American Journal of Psychology, 1972, 85, 11, 241-247.

Murch, G. M. Orientation specific colored aftereffects are classically conditioned responses. Paper presented at Sarasota, Fla., April 28-May 2, 1975.

Over, R., Long, $\mathrm{N}_{\text {. }}$ and Lovegrove, W., Absence of binocular interaction between spatial and color attributes of visual stimuli, Perception and Psychophysics, 1973, 13, pp. 534-540.

Riggs, L. Curvature as a feature of pattern vision, Science 1973, 181, pp. 1070-1072.

Skowbo, D., Gentry, T., Timney, B., and Mortant, R. B. The McCollough effect: influences of several kinds of visual stimulation on decay rate. Perception and Psychophysics, 1974, 16, pp. 47-49.

Stromeyer, C. F. Further studies of the McCollough effect, Perception and Psychophysics, 1969, 6, pp. 105-110.

Stromeyer, C. F. Edge contingent color aftereffects: spatial frequency specificity, Vision Research, 1972 12 , pp. 717-733.

Stromeyer, C. F. Contour contingent color aftereffects: retinal area specificity, American Journal of Psychology, 1972,85 , pp. 227-235.

Stromeyer, C. F. and Mansfield, J. W. Colored aftereffects produced with moving edges, Perception and Psychophysics, 1970,7 , pp. 108-114.

Teft, L. W. and Clark, F. T. The effects of stimulus den-. sity on orientation specific aftereffects of color adaptation, Psychonomic Science, 1968, 11, Pp. 265-266.

Uhlarik, J. and Osgood, A. The role of some spatial parameters of gratings on the McCollough effect. Perception and Psychophysics, 1974, 15, pp. 524-528.

Wyatt, T. J. Singly and doubly contingent aftereffects involving color orientation, and spatial frequency, Vision Research, 1974, 14, pp. 1185-1193. 


\section{APPENDIX}

I: Color Names Given by Subjects Adapted to Both Red and Green

$0^{\circ}$

no color

red

orange

pink

yellow

red

pink

orange

$40^{\circ}$

blue

yellow

yellow

yellow

yellow-green

yellow

orange

orange-yellow $10^{\circ}$

yellow

red

orange

pink

blue

red

pink

orange

$$
45^{\circ}
$$

blue

yellow

yellow

yellow

yellow-green

yellow

orange

yellow pink
200

blue

red

yellow

yeliow

yellow

red

orange

$50^{\circ}$

blue

yellow

yellow

yellow

yellow

yellow

orange

yellow

$90^{\circ}$

yeliow

yeliow

yellow

yellow

green:

yellow

orange

yellow blue

yellow

yellow

pink

yellow

yellow

orange

orange-yellow

$$
60^{\circ}
$$

blue

yellow

yellow

yellow

yellow

yellow

orange

greenish-yellow

yellow
yellow
yellow
yellow
green
yellow
orange
yellow

yellow
yellow
yellow
yellow
green
yellow
orange
yellow

yellow
yellow
yellow
yellow
green
yellow
orange
yellow

yellow
yellow
yellow
yellow
green-
yellow
orange
yellow 
II: Color Names Given by Subjects Adapted to Red Vertical (RV) or Green.Horizontal (GH) Only

$0^{\circ}$

RV

Yellow

Green-Gray

No Color

Yellow

$\mathrm{GH}$

Orange Orange-Yellow Orange Orange

$40^{\circ}$

RV

Yellow

Green-Gray

Pink

Green

$\mathrm{GH}$

Orange

Yellow

Orange

Orange

$70^{\circ}$

RV

Yellow-Green

Green

Yellow

Yellow-Green

GH

Yellow-Orange Orange-Yellow Orange

Yellow-Orange $10^{\circ}$

RV

Yellow

Green-Gray

Pink

Yellow-Green

$\mathrm{GH}$

Orange

Yellow-Orange

Orange

Orange

$45^{\circ}$

RV

Yellow

Green-Gray

Pink

Green

$\mathrm{GH}$

Orange

Orangish-Yellow

Orange

Orange

$80^{\circ}$ :

$R \dot{V}$

Yellow

Green-Yellow

Yellow

Yellow-Green

GH

Orange

Orange-Yellow

Orange

$50^{\circ}$

RV

Yellow-Orange Yellow

Green...... Green

No Color

Pink

Yellow-Green Yellow-Green

$\mathrm{GH}: \quad \mathrm{GH}$

Trange Trange

Orangish-Yellowyellow-Orange

Red-Orange

Orange

Red-Orange

Yellow-Orange

RV

Green-Yellow

Pink

Green

$\mathrm{GH}$

Orange

Yellow

Orange
No Color 
Debbi Davis 